\title{
Application of Improved RFID Anti - Collision Algorithm in Library Management
}

\author{
Huijie Guo ${ }^{1}$ \\ China Pharmaceutical University, Nanjing, Jiangsu, 211198, China \\ E-mail:ghj20560163.com
}

\section{Lei $\mathrm{CaO}^{2}$}

China Pharmaceutical University, Nanjing Jiangsu, 211198, China

E-mail:caolei@cpu.edu.cn

\section{Jianzhen $\mathbf{X u}^{\mathbf{3}}$}

China Pharmaceutical University, Nanjing Jiangsu, 211198, China

E-mail:xujz@cpu. edu. cn

Radio Frequency identification (RFID) has integrated a wide range of applications for the identification and traceability of benefits . The tag collision remains a major drawback in radiofrequency identification systems, especially in large-scale ones. Thus it is critical to establish algorithms on the basis of hardware to realize accurate and efficient identification to optimize the system.Based on the analysis of query tree and dynamic frame time slot ALOHA algorithm, a prioritized layered RFID anti-collision algorithm is presented as an improved method to solve these problems for the library circumstance. The query count, transmission delay, throughput rate and other performance indicators are compared between those algorithms to evaluate the effectiveness of this algorithm. As indicated by the simulation results, it has less collision probability than DFSA and less queries than QT with transmission delay increased to a certain extent, showing better performance and scalability in the large amount of tags like the library management.

\section{CENet2017}

22-23 July, 2017

Shanghai China

\footnotetext{
${ }^{1}$ undergraduate, assistant of library director,research interests: management and application of digital library.

${ }^{2}$ master, research interests: network security and information security, large network integration and management

${ }^{3}$ professor, research interests: interconnections between main research direction is computer communication and network technology, management information system application and development.
} 


\section{Introduction}

Radio frequency identification (RFID), as one of the most promising wireless communication technologies, has been widely used in many intelligent management fields. Generally, an RFID system could identify multiple tags simultaneously within the working scope; however, an important issue of this system is the interference of RF signal in the wireless channel when a large amount of tags response to the reader's query, then the reader cannot deal with these requests completely in a short time and part of them are rejected, leading to the efficiency reduction of RFID system.

There are a variety of ways to anti-collision in the hardware of RFID systems. TDMA technology is used extensively with simple structure and low energy consumption, strong operation [1], etc. The existing anti-collision mechanisms under TDMA technology generally adopt either probabilistic ALOHA-based algorithms or the deterministic tree-based algorithms.

\section{RFID Anti-collision Algorithm}

\subsection{Algorithms based on Aloha Mechanism}

The tags send their own ID information randomly, and communication between readers and tags goes normally when single tag request, if multiple tags respond synchronized, the RF signal would be overlapped, some tags would receive a negative acknowledgment frame and wait for another period of time to resend [2]. The frame-slotted ALOHA (FSA) Algorithm and dynamic frame time slot ALOHA (DFSA) algorithm are proposed to reduce the collisions further than in time-slots (SA) algorithm which avoids local collision.

The DFSA can change the frame length adaptively than the FSA so that the system can maintain a higher through-put at all stages; however, the maximum frame length less than $256[3]$ constraints on account of hardware .

\subsection{Algorithm Based on Binary Tree}

The tree-based algorithms on binary are most widely adopted in RFID system. Those methods partition RFID tags by dividing ID sequences into two subsets, 1 and 0 , then traverse from the subset 0 until all tags are read[4]. Besides, there are also two common algorithms, the query tree search (QT) algorithm and the tree splitting (TS) algorithm.

A new query string is formed by adding 1 bit each time and all tags in the recognition scope can be readin the QT algorithm . However, with the tag number increasing, the entire identification cycle is extended, the operation process is more complex and the energy consumption increases sharply because of idle binary code.

\section{Algorithm Based on Priority Control}

An RFID system is required to enhance the progressive realization of library management and service processes [5] because of great workload of books which also cause mass tag collision. This paper proposes a prioritized and layered ALOHA (PLA) algorithm under library management environment. The PLA adds the location information in the book tag such as reading room, and other data encoded with binary bits number, then tags be divided into 
multiple groups and layers based on hierarchical architecture, then the groups are prioritized dynamically within the layers to adjust the matching order by a mathematical model [6].

\subsection{Priority Reference Model}

(1) The format of RFID book location information and ID coding.

The tag location data occupy four bytes including reading room number, bookshelf number, layer number and priority number in the layer, be allotted to $8,12,4,8$ and 8-bits respectively. The location information format is shown in Table 1.

\begin{tabular}{|l|l|l|l|l|l|}
\hline Data Information & $\begin{array}{l}\text { Reading Room } \\
\text { Number }\end{array}$ & $\begin{array}{l}\text { Bookshelf } \\
\text { Number }\end{array}$ & $\begin{array}{l}\text { Layer } \\
\text { Number }\end{array}$ & $\begin{array}{l}\text { Priority Number in } \\
\text { Layer }\end{array}$ & Label ID \\
\hline Length (bit) & 8 & 12 & 4 & 8 & 8 \\
\hline Data Range & $0^{\sim} 255$ & $0^{\sim} 1023$ & $0^{\sim} 15$ & 0 & 0255 \\
\hline
\end{tabular}

Table 1:Book Tags Store Segment Information

(2) Layers and Groups

The structure is layered according to the tag quantity in the location range. Tags are divide into several groups in each layer. There are three layers can be grouped in PLA, reading room, bookshelves and bookshelf layer under this situti. The tags involve the following types:

(1) The leader tag. It has the highest priority in its group.

(2) Root.The highest priority member in the system.

(3) Group Member (GM). It communicates and connects with each other.

(3) Priority mechanism

The leader tag is always visited preferentially. Here, a layered and prioritized model shown in Fig. 1 is developed to make selection from the existing members as leaders.

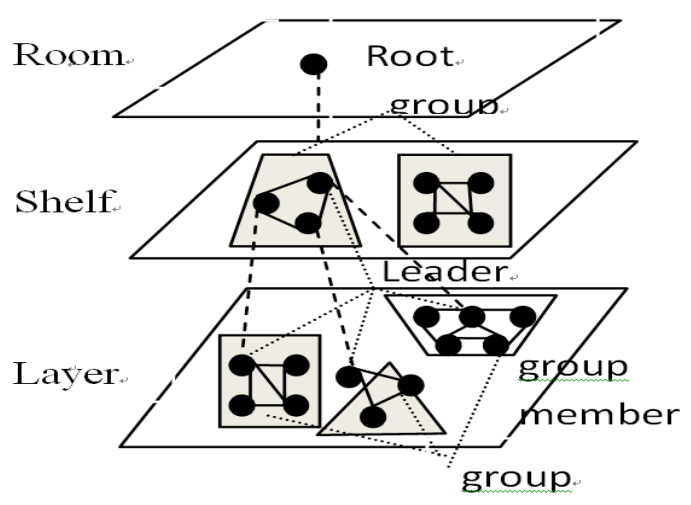

Figure 1: Priority Reference Model under library management environment

\subsection{Priority Mathematical Model}

In this priority model, the activity $(A)$ of book tags can be calculated from the times recognized by readers. The duration (Dur) time that a book being read is given by the recorded interval tag departure from the shelf. The duplicate ratio $(D R)$ represents the quantity of the same books as distinguished by ISBN number [7], on count of the number of readers' borrow times, their values and other factors. $P(A, A D, D R)$ indicates the priority of tags in their groups and are also recorded in the database of the RFID system.

If $n$ duplicate books are placed on the same bookshelf layer, the activity of duplicates in a period time is shown by the following matrix which consists of lend and retubooks, $\{x[1], x$ 
$[2], x[3], \ldots, x[k], \ldots, x[n]\}$ ( $x$ represents the lending or return times, $n$ is the amount of duplicates, $l \leq k \leq n)$, then we will have:

Total lend times: $\quad L=1[1]+1[2]+\cdots+1[\mathrm{k}]+\cdots+1[\mathrm{n}]$

Total return times: $\mathrm{R}=\mathrm{r}[1]+\mathrm{r}[2]+\ldots+\mathrm{r}[\mathrm{k}]+\ldots+\mathrm{r}[\mathrm{n}]$

Then the activity $A=\frac{L}{R}$ can be expressed as:

$$
A=\frac{l[1]+l[2]+\ldots+l[k]+\ldots+l[n]}{r[1]+r[2]+\ldots+r[k]+\ldots r[n]} \text { note: }(\mathrm{A} \geq 1)
$$

The duplicate ratio usually lies between the number of duplicates and the variety of books. For one kind of book with $n$ duplicates $n(r, s, l)$.

So, the duplication ratio is defined as: $\quad D R=\frac{1}{n(r, s, l)}$

The time book reserved by the reader $t(r)$ can be calculated according to the time interval resders keeping and a buffer factor $\varepsilon$ is set to record the half-baked interval.

For one kind of book, the total duration is calculated as: $D u r=\sum_{r=1}^{R} t(r)+\varepsilon$

The priority of the book tags can be defined as: $P_{(r, s, l)}=\frac{D u r}{A} \bullet D R$

If there are Num books and $m$ kinds of books within managing scope, $\operatorname{Dur}(N)$ and $A(m)$ represent the the reading duration and activity of those books, so the priority $P(A, D u r, D R)$ of can be concluded as:

$$
P_{(r, s, l)}=\frac{\left[\sum_{r=1}^{R} t(r)+\varepsilon\right] \bullet \sum_{k=1}^{n} r[k]}{\sum_{k=1}^{n} l[k] \bullet n_{(r, s, l)}}
$$

\section{Performance Analyses}

\section{4..1Query Counts in QT and PLA}

The tag data collision occurs mainly on the bifurcate nodes of the binary tree in the QT algorithm. If $N$ tags are waiting for identification, the maximum depth of complete binary tree means $[\log N / \log 2]+1$ in the worst case, then the total query counts is:

$$
Q C_{1}=2^{[\log N / \log 2]+1}-1=2 * N-1
$$

The PLA algorithm is used to classify the label subsets in hierarchical order. Since the groups of $\mathrm{N}$ tags have different mapping relations, the query counts in the PLA algorithm is related to the logical order of tags.If $\mathrm{N}$ tags in order under the worst case, then:

$$
Q C_{2}=N \cdot \sum_{1}^{k} \log _{g(k)} L_{k}
$$

Where, $k$ represents the number of logical layers, ${ }^{g}(k)$ represent the number of intra-layer packets and the length of each field is represented by $L_{(k)}$.

If the logical distribution probability density of tags is $p, t_{(k)}$ indicates random queriesis:

$$
Q C_{3}=N \cdot \sum_{1}^{k} \sum_{1}^{t(k)} \log _{t(k)} L_{k} \quad \text { note: } t_{(k)}=p \cdot g_{(k)}
$$

The length of the tag UID in this model is 40 bit, UHF RFID systems are adopted in library management which works in $905-925 \mathrm{MHZ}$. Assume the frequency of noise error is 
$1 \mathrm{KHZ}$ [8], the read rate under this sampling frequency can reach $\mathrm{v}=80 \mathrm{kbit} / \mathrm{s}$ and satisfied the normal distribution, the query counts as shown in Fig.2, the PLA reduces query times significantly when compared to the QT algorithm, and there are less queries if the tags with higher priority in the model. Besides, the gap of query count between tags with different priorities will decrease with the tag number increasing.

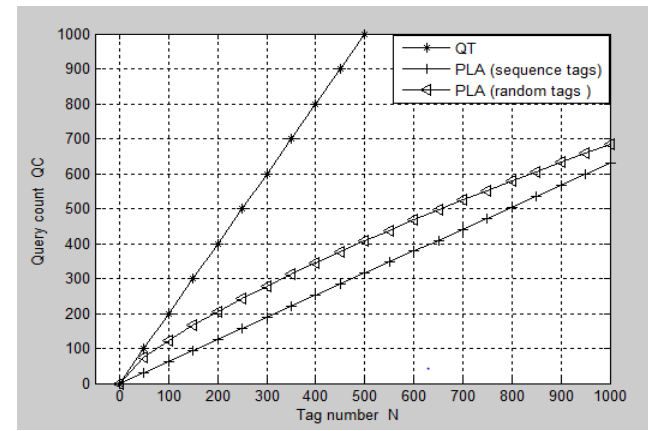

Figure 2: The Query Counts in QT,Sequence or Random Tags in PLA

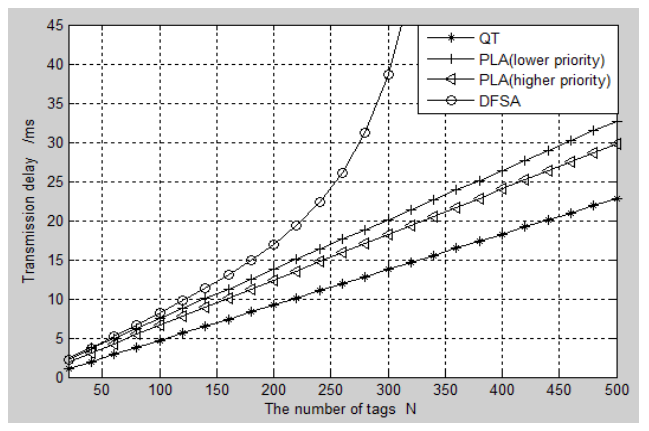

Figure 3: The Transmission Delay of N Tag in the PLA, QT and DFSA

\subsection{Transmission Delay in QT, PLA and DFSA}

The transmission delay includes the information reading and query disposing in QT and PLA. If there are $N$ tags waiting for confirmation within the readers' working scope, assuming the time to read one tag is $T_{0}$.then the transmission delay is:

$$
T_{Q T}=N \cdot T_{0}+t_{q} \log _{2} N
$$

For tags' locations is represented by $\operatorname{Tag}(r, s, l, p)$ in PLA, thus the delay to read ID information of leader tag is:

$$
T_{L T}=t_{r_{0}}+t_{s_{0}}+t_{I_{0}}+t_{p_{0}}+T_{0}=t_{L L}+T_{0}
$$

Here, $T_{0}$ represents the time to read UID information in a tag, $t_{x_{0}} t_{s_{0}} t_{l_{0}} t_{p_{0}}$ represent the delay to explore each layer respectively, $t_{L L}$ represents the length of time to match four layers.

When $N$ tags wait to be read, the intervals for each step are assigned $A, B, C$, and $D$ the maximum delay required is:

$$
T_{N_{\max }}=t_{L T}+r_{\max } A+s_{\max } B+l_{\max } C+p_{\max } D+N * T_{0}
$$

In the DFSA, the delay of the transmitting $\mathrm{N}$ tags includes reading and retransmission, because of collision. Assume the retransmission of timeout period tags start after $\mathrm{R}^{*}{ }_{T_{\mathrm{o}}}$, the maximum duration of this process is $\mathrm{k} * T_{\mathrm{o}}$, the frame length is $\mathrm{FL}=256$, then the average time of ${ }_{N_{R}}$ collided frame retransmission is:

$$
T_{C}=T_{0}\left[1+R+N_{R}\left(R+\frac{k+1}{2}\right)\right]
$$

The probability of tag collision in DFSA is:

$$
P_{C}=\frac{N}{F L\left(1-\frac{1}{F L}\right)^{N-1}}
$$

So, the delay of transmitting $\mathrm{N}$ labels in the DFSA algorithm is given by (4.7) and (4.8)is : 


$$
T_{D F S A}=N \cdot T_{0}\left(1-\frac{1}{L}\right)^{N}+T_{0}\left[1+R+N_{R}\left(R+\frac{K+1}{2}\right)\right]
$$

The comparison of transmission delay between them is shown inFig. 3.From the results of analysis in Fig. 3, the transmission delay of DFSA will greatly increase with tag collision occurs. Both PLA and QT maintain a small delay relatively, and the delay in QT has a smaller growth rate because the subsets are divided into smaller unit.

\subsection{Throughput of PLA and DFSA}

The transmission delays differ greatly in various priority locations.Here, the average amount of data $G$ in a certain period time in PLA can be defined as:

$$
G=\sum_{1}^{N} T_{(N)} \bullet \frac{\sigma}{t}
$$

Where $n=1,2,3 \ldots$ is the number tags in the system, $\sigma$ indicates the amount of data in a tag, $T_{(N)}$ indicates the delay to transmit a tag, $t$ for a period of time.

If $n_{\max }$ tags can be contain in average data packet capacity per unit time, the average throughput of PLA can be expressed as:

$$
s=\left\{\begin{array}{lr}
\sum_{1}^{N} T_{(N)} \cdot \frac{\sigma}{t} & 0 \leq N<N_{\max } \\
\sum_{1}^{N_{\max }} T_{\left(N_{\max }\right)} \cdot \frac{\cdot \sigma}{t} & N \geq N_{\max }
\end{array}\right.
$$

Denote the time slots occupied successfully be I, the idle or collision time slots be F[9] in the DFSA algorithm. If the frame length is FL, the probabilities that tags in I or F slots are:

$$
\begin{gathered}
P_{I}=\frac{N}{F L\left(1-\frac{1}{F L}\right)^{N-1}} \\
P_{F}=1-P_{I}=1-\frac{N}{F L\left(1-\frac{1}{F L}\right)^{N-1}}
\end{gathered}
$$

The system throughput in the DSFA algorithm is only related to the number of time slots occupied successfully. For the number of I slots, the mathematical expectation is:

$$
E(I)=N\left(1-\frac{1}{F L}\right)^{N-1}
$$

Then the system throughput $\mathrm{S}$ per unit time can be expressed as:

$$
S=\frac{E(I)}{L}=\frac{N}{F L}\left(1-\frac{1}{F L}\right)^{N-1}
$$

For the maximum value of the throughput curve $\mathrm{S}(\mathrm{N}, \mathrm{FL})$, partial derivative on $\mathrm{N}$ in the above formula is :

$$
\frac{d S}{d N}=\frac{1}{F L}\left(1-\frac{1}{F L}\right)^{x-1}\left[1+N I n\left(1-\frac{1}{F L}\right)\right]
$$

If tags in a large number, $\frac{d S}{d N}=0$ can be simplified by Taylor series as:

$$
F L=\frac{1+1 / N}{1+1 /(N+1)}=N+1 \quad(\mathrm{~N}>>1)
$$

Throughput analysis between DSFA and PLA algorithm as shown in Fig. 4.

As shown in Fig. 4, compared with the DFSA algorithm, it can improve the throughput in a certain extent when the number of packets communicated per unit time is less than the amount 
of saturated packets.The DFSA achieves the maximum throughput when $\mathrm{N}=\mathrm{L}$, then the throughput rate decline gradually due to the collision probability increasing, and the throughput increases in PLA until accommodating the maximum tags.

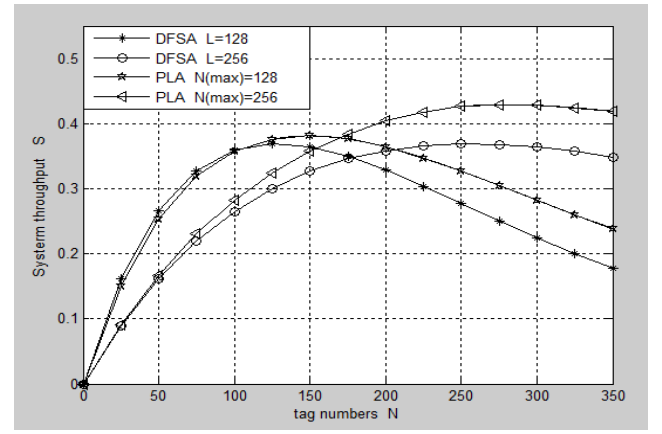

Figure 4: Throughput Analysis between DSFA and PLA Algorithm

\section{Conclusion}

On the basis of the analysis of QT and DFSA algorithms, this algorithm can adjust the order to identification, the query times is reduced under the acceptable transmission delay and the system throughput is improved to a certain extent. The simulation results show the superiority and feasibility of PLA algorithm, although the complexity of the priority model will increase the energy consumption of the overall system, which needs to be further improved. PLA features a certain reference value to improve the efficiency of RFID system in term of large-scale management.

\section{References}

[1] Yue Ke-qiang. Research on Multi-tag Anti-collision Algorithms for RFID Systems and Applica-tion.[D]. Zhe Jiang: Zhe Jiang University, 2014:7-9.

[2] Klair D K, Chin K W, Raad R. A survey and tutorial of RFID anti-collision protocol. [J]. IEEE

Communication Surveys and Tutorial, 2010, 12(03):400-421.

[3]VOGT H. Multiple object identification with passive RFID tags [C]//ICSMC 2002: IEEE Interna -tional Conference on System .Man and Cybernetics. Zurich Switzerland: IEEE, 2002:98-113.

[4]Liu Xiao-gang, Song Gao-jun. Overview of Anti-collision Algorithm in RFID. [J]. Wireless

Internet Technology, 2015(03): 26-27.

[5]Yao Yuan. The Application and study of RFID Technology in University Library. [D]. Shang Hai: Institute of Information Science and Technology Shanghai Jiao Tong University, 2011:52-54.

[6]Xu Jian-zhen. Study ofThe Key Technologies of The Application Layer Multicast. [D]. Nan Jing: Nan Jing University. 2009: 33-40.

[7]Tian Lei .Dupincation Control in Book Acquisition. [J].The Library Journal of Shandong, 2016(02):60-63.

[8]Sun zhiyong.Design and Realization of Digital Signal Processing Modules in RFID Sysem. [D]. Hangzhou :Hangzhou Dianzi University.2013:16-19.

[9]Gong Tengfei, Research of Dynamic Frame Slotted Aloha and Query Tree Anti-collision Algorithm in RFID System. [D].Chong Qing: Chong Qing University Of Posts And Tele commnuicaion. 2016:14-24. 